Research Article

\title{
Sum Squeezing of the Field Amplitude in Frequency Upconversion Process
}

\author{
Dilip Kumar Giri $\mathbb{D}^{1}$ and Binod Kumar Choudhary ${ }^{2}$ \\ ${ }^{1}$ University Department of Physics, Binod Bihari Mahto Koyalanchal University, Dhanbad, India \\ ${ }^{2}$ University Department of Physics, Vinoba Bhave University, Hazaribag, India \\ Correspondence should be addressed to Dilip Kumar Giri; dilipkumargiri@gmail.com
}

Received 4 October 2019; Revised 18 December 2019; Accepted 31 December 2019; Published 1 February 2020

Academic Editor: Paramasivam Senthilkumaran

Copyright (c) 2020 Dilip Kumar Giri and Binod Kumar Choudhary. This is an open access article distributed under the Creative Commons Attribution License, which permits unrestricted use, distribution, and reproduction in any medium, provided the original work is properly cited.

\begin{abstract}
Sum squeezing of the field amplitude is studied in the nondegenerate and degenerate frequency upconversion process under the short interaction time. It is shown that sum squeezing can be converted into normal squeezing via sum-frequency generation in the nondegenerate frequency upconversion process, while the amplitude-squared squeezing of the fundamental mode directly changed into the squeezing of the harmonic in the degenerate frequency upconversion process. All reachable conditions of uncorrelated modes for obtaining a sum squeezing in two modes and its dependence on the squeezing of individual field modes are investigated. It is found that the squeezed states are associated with large number of pump photons. It is also confirmed that the higher-order squeezing (sum squeezing) is directly associated with coupling of the field and interaction time.
\end{abstract}

\section{Introduction}

Over the past years, the squeezing [1-6] in quantized electromagnetic fields has received a great deal of attention because of its wide applications in many branches of science and technology, especially for low quantum fluctuations [7-9] having potential application in optical telecommunication [10], quantum cryptography [11, 12], and others. It is a consequence of uncertainty relations. A state is squeezed when the quantum fluctuation (amplitude noise or phase noise) in one variable is reduced below the symmetric limit at the expense of the increased quantum fluctuation in the conjugate variable such that the Heisenberg uncertainty relation is not violated. It has been focused on theoretical as well as experimental evidences of squeezed states in various nonlinear optical processes, such as harmonic generation [13-16], multiphoton processes [17-20], and Raman and hyper-Raman processes [21-25]. Hong and Mandel [26, 27] and further Hillery [28] have introduced the notion of higher-order squeezing of quantised electromagnetic field as generalization of the much discussed normal squeezing and followed by [29] for improving the performance of many optical devices. Squeezing and photon statistical effect of the field amplitude in optical parametric processes and in Raman and hyper-Raman scattering has been reported by Perina et al. [30] in which it is demonstrated that squeezing accompanies antibunching very often, but not always. In some cases, squeezing may occur and antibunching may not and vice versa. Kim and Yoon [31] have also studied higherorder sub-Poissonian statistics of light and pointed out the nonclassical measure of the higher-order sub-Poissonian photon statistics of the number state is half as same as that of the known lowest order. Recently, Prakash and Mishra $[32,33]$ have also reported the higher-order sub-Poissonian photon statistics and their use in detection of Hong and Mandel squeezing and amplitude-squared squeezing. Another type of seminal paper on higher-order squeezing, called sum and difference squeezing, was proposed by Hillery [34] for the two modes which are in fact the simplest versions of multimode higher-order squeezing. These concepts have been generalized to include three modes for sum and difference squeezing [35-37] as well as an arbitrary number of modes for sum and difference squeezing [38-40]. Furthermore, more recently, Prakash and Shukla [41], 
Mukherjee et al. [42], and Mukherjee et al. [43] have also studied and reported about sum and difference squeezing and their detections in some nonlinear optical processes.

The objective of this paper is to study the sum squeezing in the nondegenerate and degenerate frequency upconversion process under the short-time scale based on a fully quantum approach. It is a higher-order squeezing of the radiation field to achieve significantly larger quantum noise reduction. Since higher-order squeezing is the higher powers of the field amplitude, which is directly associated with the large numbers of photons that make it possible to achieve significantly larger noise reduction than ordinary squeezing. This motivates us to study sum squeezing (higher-order) in the frequency upconversion process in the line of seminal paper [34]. The paper is organized as follows. Section 2 gives the definition of one- and two-mode higher-order squeezing. Sum squeezing of the field amplitude in the nondegenerate frequency upconversion process is investigated in Section 3. Detection of sum squeezing of two-mode field in this process is also studied in Section 3. In Section 4, sum squeezing of the field amplitude in the degenerate frequency upconversion process is studied and the relation between sum squeezing and amplitude-squared squeezing is established. Finally, we conclude the paper in Section 5.

\section{Definition of One- and Two-Mode Higher- Order Squeezing}

Suppose a single mode of radiation field having frequency $\omega_{a}$ with creation and annihilation operators $a^{\dagger}$ and $a$, respectively, and defining amplitude-squared squeezing in terms of operators $Y_{1}$ and $Y_{2}$ given by

$$
\begin{aligned}
& Y_{1}=\frac{1}{2}\left(A^{2}+A^{\dagger 2}\right), \\
& Y_{2}=\frac{1}{2 i}\left(A^{2}-A^{\dagger 2}\right),
\end{aligned}
$$

where $A=a \exp \left(i \omega_{a} t\right)$ and $A^{\dagger}=a^{\dagger} \exp \left(-i \omega_{a} t\right)$ are slowly varying operators.

Equation (1) obeys the commutation relation

$$
\left[Y_{1}, Y_{2}\right]=i\left(2 N_{A}+1\right)
$$

where $A^{\dagger} A=N_{A}$ is the number operator.

Relation (2) leads to the uncertainty relation $(\hbar=1)$ :

$$
\Delta Y_{1} \Delta Y_{2} \geq\left\langle N_{A}+\frac{1}{2}\right\rangle \text {. }
$$

Equation (3) exists amplitude-squared squeezing if it follows the condition:

$$
\left(\Delta Y_{j}\right)^{2}<\left\langle N_{A}+\frac{1}{2}\right\rangle,
$$

where $j=1$ or 2 and $\Delta Y_{1}$ and $\Delta Y_{2}$ are the uncertainties in the quadrature operators $Y_{1}$ and $Y_{2}$, respectively.

A quantum state is amplitude-squared squeezed in the $Y_{1}$ direction if $\left(\Delta Y_{1}\right)^{2}<\left\langle N_{A}+(1 / 2)\right\rangle$ and is amplitudesquared squeezed in the $Y_{2}$ direction if $\left(\Delta Y_{2}\right)^{2}<\left\langle N_{A}+\right.$ $(1 / 2)\rangle$.
Now, for two modes having frequency $\omega_{a}$ and $\omega_{b}$ with creation (annihilation) operators $a^{\dagger}(a)$ and $b^{\dagger}(b)$, let us introduce two operators which correspond to real and imaginary parts of the product of the field amplitudes as

$$
\begin{aligned}
& W_{1}=\frac{1}{2}\left(A B+A^{\dagger} B^{\dagger}\right), \\
& W_{2}=\frac{1}{2 i}\left(A B-A^{\dagger} B^{\dagger}\right),
\end{aligned}
$$

where $A=a \exp \left(i \omega_{a} t\right)$ and $B=b \exp \left(i \omega_{\mathrm{b}} t\right)$ are slowly varying operators.

Equations (5) and (6) follow the commutation relation

$$
\left[W_{1} W_{2}\right]=\frac{i}{2}\left(N_{A}+N_{B}+1\right)
$$

and satisfy the uncertainty relation $(\hbar=1)$ :

$$
\Delta W_{1} \Delta W_{2} \geq \frac{1}{4}\left\langle N_{A}+N_{B}+1\right\rangle
$$

where $N_{A}=A^{\dagger} A$ and $N_{B}=B^{\dagger} B$ are the number operators.

Sum squeezing in the $W_{j}$ direction exists if

$$
\left(\Delta W_{j}\right)^{2}<\frac{1}{4}\left\langle N_{A}+N_{B}+1\right\rangle,
$$

where $j=1$ or 2 and $\Delta W_{1}$ and $\Delta W_{2}$ are the uncertainties in the quadrature operators $W_{1}$ and $W_{2}$, respectively.

A state is sum squeezed in the $W_{1}$ direction if $\left(\Delta W_{1}\right)^{2}<$ $(1 / 4)\left\langle N_{A}+N_{B}+1\right\rangle$ and is sum squeezed in the $W_{2}$ direction if $\left(\Delta W_{2}\right)^{2}<(1 / 4)\left\langle N_{A}+N_{B}+1\right\rangle$.

\section{Sum Squeezing in the Nondegenerate Frequency Upconversion Process}

Frequency upconversion, shown in Figure 1, is a three-wave interaction nonlinear optical phenomenon, in which two input photons (a signal and a pump photon) at different frequencies, $\omega_{\mathrm{a}}$ and $\omega_{\mathrm{b}}$, annihilate and another photon at their sum frequency, $\omega_{c}$, is simultaneously generated in the nonlinear optical medium.

It serves as basic building blocks for the implementation of quantum optical experiments. By using this technique, near-infrared light can be converted to light in the visible or near-visible range and therefore detected by commercially available visible detectors with high efficiency and low noise. It can be realized in nonlinear crystals, but the relatively low conversion efficiency requires a high-power laser or a resonant cavity [44]. This model is chosen to make a realistic one, and our theoretical discussions hold for all similar models.

The Hamiltonian of this process may be written as $(\hbar=1)$

$$
H=\omega_{a} a^{\dagger} a+\omega_{b} b^{\dagger} b+\omega_{c} c^{\dagger} c+g\left(a b c^{\dagger}+a^{\dagger} b^{\dagger} c\right),
$$

where $a^{\dagger}(a), b^{\dagger}(b)$, and $c^{\dagger}(c)$ are the creation (annihilation) operators of the $A, B$, and $C$ modes, respectively, and $g$ is the coupling constant between the two modes of the order of $10^{2}-10^{4}$ per second and is proportional to the nonlinear susceptibility of the medium as well as the complex 


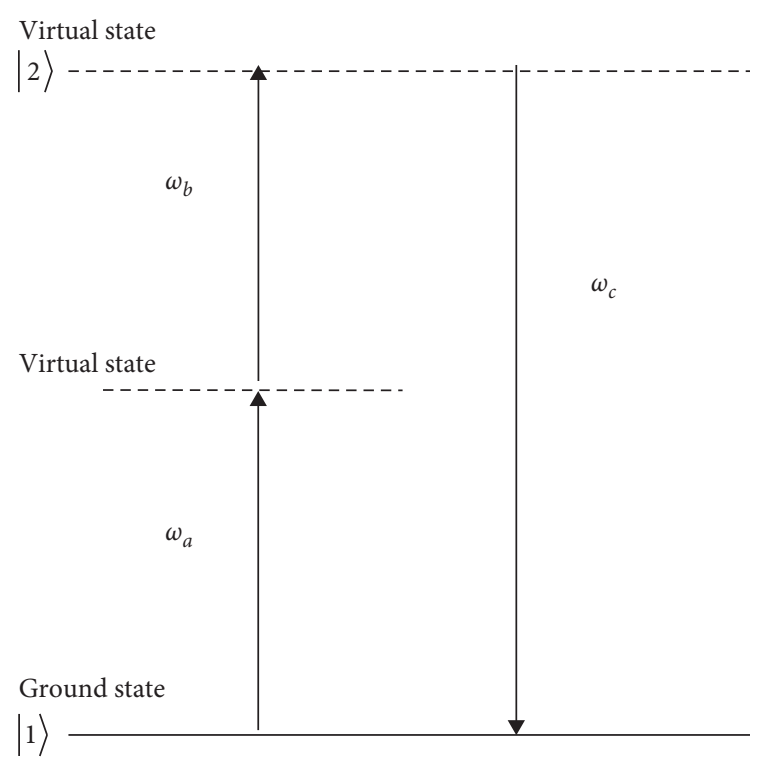

Figure 1: Energy level diagram of the frequency upconversion process.

amplitude of the pump field $[4,30,45]$. However, to take care of complex $g$, we have used $|g|^{2}$ in the place of $g^{2}$ [4].

Using the interaction Hamiltonian of equation (10) in the coupled Heisenberg equation of motion,

$$
\dot{A}(t)=\frac{\partial A(t)}{\partial t}+i[H, A(t)], \quad \hbar=1,
$$

where the dot denotes time derivative.

Equation (11) leads to

$$
\begin{aligned}
& \dot{A}=-i g B^{\dagger} C, \\
& \dot{B}=-i g A^{\dagger} C, \\
& \dot{C}=-i g A B,
\end{aligned}
$$

where $A, B$, and $C$ are slowly varying operators, which are defined by $A=a \exp \left(i \omega_{a} t\right), \quad B=b \exp \left(i \omega_{b} t\right)$, and $C=c$ $\exp \left(i \omega_{c} t\right)$, with the relation $\omega_{a}+\omega_{b}=\omega_{c}$. The operators $A(t)$ and $A^{\dagger}(t)$ induce a slower dependence on time as compared to fast variation during the interaction between modes.

The system evolution during a short period of time is practically relevant because the actual interaction is in fact very short. Hence, the interaction time is taken to be short, of the order of $10^{-10} \mathrm{~s}$, and a nanosecond or picosecond pulse laser can also be used as the pump field for time resolved measurements $[4,44]$. For real physical situation in the short-time scale $g t \ll 1\left(g t \sim 10^{-6}\right)$, the expectation value of mean pump photon numbers is very large $\left(\left\langle A^{\dagger} A\right\rangle=|\alpha|^{2} \gg 1\right)$ and it is possible to obtain much simpler approximate analytical formulas describing the variances [4].

Using Taylor's expansion on $A(t)$ as $A(t)=A(0)+$ $t \dot{A}(0)+\left(t^{2} / 2 !\right) \ddot{A}(0)+\ldots \ldots$ and keeping terms up to second order in $t$, we have

$$
A(t)=A+t\left(-i g B^{\dagger} C\right)+\frac{t^{2}}{2 !}\left[-i g\left(\dot{B}^{\dagger} C+B^{\dagger} \dot{C}\right)\right],
$$

where $\dot{A}=-i g B^{\dagger} C$ and $\ddot{A}(0)=\left[-i g\left(\dot{B}^{\dagger} C+B^{\dagger} \dot{C}\right)\right]$ and after simplification, we get

$$
\begin{aligned}
& A(t)=A+t\left(-i g B^{\dagger} C\right)+\frac{t^{2}}{2 !}\left[-i g\left(i g A C^{\dagger} C\right)+B^{\dagger}(-i g A B)\right] \\
& \text { or } A(t)=A+t\left(-i g B^{\dagger} C\right)+\frac{g^{2} t^{2}}{2 !}\left[C^{\dagger} C-B^{\dagger} B\right] A \\
& \text { or } A(t)=A-i g t B^{\dagger} C+\frac{1}{2}|g|^{2} t^{2}\left(N_{C}-N_{B}\right) A,
\end{aligned}
$$

where $C^{\dagger} C=N_{C}$ and $B^{\dagger} B=N_{B}$.

$$
A^{\dagger}(t)=A^{\dagger}+i g t B^{\dagger} C+\frac{1}{2}|g|^{2} t^{2}\left(N_{C}-N_{B}\right) A^{\dagger} .
$$

Similarly,

$$
\begin{gathered}
B(t)=B-i g t A^{\dagger} C+\frac{1}{2}|g|^{2} t^{2}\left(N_{C}-N_{A}\right) B^{\dagger}, \\
B^{\dagger}(t)=B^{\dagger}-i g t A C^{\dagger}+\frac{1}{2}|g|^{2} t^{2}\left(N_{C}-N_{A}\right) B .
\end{gathered}
$$

Also,

$$
C(t)=C-i g t A B-\frac{1}{2}|g|^{2} t^{2}\left(N_{A}+N_{B}+1\right) C,
$$

$$
C^{\dagger}(t)=C^{\dagger}-i g t A^{\dagger} B^{\dagger}-\frac{1}{2}|g|^{2} t^{2}\left(N_{A}+N_{B}+1\right) C^{\dagger} .
$$

Let us examine squeezing in the $C$ mode, and we define two general quadrature components:

$$
\begin{aligned}
& X_{1 C}(t)=\frac{1}{2}\left[C(t)+C^{\dagger}(t)\right], \\
& X_{1 C}(t)=\frac{1}{2}\left[C(t)-C^{\dagger}(t)\right] .
\end{aligned}
$$

Using equations (18) and (19) in equations (20) and (21), we obtain

$$
\begin{aligned}
& X_{1 C}(t)=X_{1 C}+|g| t W_{2}-\frac{1}{2}|g|^{2} t^{2}\left(N_{A}+N_{B}+1\right) X_{1 C}, \\
& X_{2 C}(t)=X_{2 C}-|g| t W_{1}-\frac{1}{2}|g|^{2} t^{2}\left(N_{A}+N_{B}+1\right) X_{2 C} .
\end{aligned}
$$

At $t=0$, for uncorrelated modes, we get

$$
\begin{aligned}
{\left[\Delta X_{1 C}(t)\right]^{2}=} & \left(\Delta X_{1 C}\right)^{2}+|g|^{2} t^{2} \\
& \cdot\left[\left(\Delta W_{2}\right)^{2}-\left\langle N_{A}+N_{B}+1\right\rangle\left(\Delta X_{1 C}\right)\right]^{2},
\end{aligned}
$$

$$
\begin{aligned}
{\left[\Delta X_{2 C}(t)\right]^{2}=} & \left(\Delta X_{2 C}\right)^{2}+|g|^{2} t^{2} \\
& \cdot\left[\left(\Delta W_{1}\right)^{2}-\left\langle N_{A}+N_{B}+1\right\rangle\left(\Delta X_{2 C}\right)\right]^{2}
\end{aligned}
$$

If initially the $C$ mode is in a coherent state, then 


$$
\left(\Delta X_{1 C}\right)^{2}=\left(\Delta X_{2 C}\right)^{2}=\frac{1}{4}
$$

and equations (24) and (25) reduce to

$$
\begin{aligned}
& {\left[\Delta X_{1 C}(t)\right]^{2}-\frac{1}{4}=|g|^{2} t^{2}\left[\left(\Delta W_{2}\right)^{2}-\frac{1}{4}\left\langle N_{A}+N_{B}+1\right\rangle\right],} \\
& {\left[\Delta X_{2 C}(t)\right]^{2}-\frac{1}{4}=|g|^{2} t^{2}\left[\left(\Delta W_{1}\right)^{2}-\frac{1}{4}\left\langle N_{A}+N_{B}+1\right\rangle\right] .}
\end{aligned}
$$

Equations (27) and (28) establish the relation between sum squeezing and normal squeezing in the frequency upconversion process. We find that if the input state is sum squeezed in the $W_{2}$ or $W_{1}$ direction, then normal squeezing will occur in the $X_{1 C}$ or $X_{2 C}$ direction, respectively. This result suggests a method for detection of nonclassical properties of radiation in the frequency upconversion process.

We plot a graph (Figures 2 and 3) between left-hand side of equations (27) and (28) say $S_{\mathrm{S}}$ and $S_{\mathrm{S}}^{\prime}$, respectively, versus $|g t|^{2}$ with typical values $\left(\Delta W_{2}\right)^{2}=\left(\Delta W_{1}\right)^{2}=(1 / 4)$ so that it could satisfy equation (9).

The steady fall of the curves infers that the sum squeezing exists and responses nonlinearly to the number of pump photons. It shows that when $|\alpha|^{2}$ increases, the degree of sum squeezing also increases, i.e., $S_{\mathrm{S}}$ is getting more negative. This confirms that the squeezed states are associated with large number of pump photons. It also confirms that the higher-order squeezing (sum squeezing) is directly associated with the coupling of the field and interaction time. Hence, optimum squeezing can be realized in short-time scale.

Comparing Figures 2 and 3, we inferred that the depth of nonclassicality is increasing with an increase of $|\beta|^{2}$. Hence, it is inferred that a higher multiphoton absorption process is suitable for generation of optimum squeezed light.

It is also of interest to study sum squeezing in the $\mathrm{C}$ mode as a function of time; we define the quadrature operators as follows:

$$
\begin{aligned}
& W_{1 C}(t)=\frac{1}{2}\left[A(t) B(t)+A^{\dagger}(t) B^{\dagger}(t)\right], \\
& W_{2 C}(t)=\frac{1}{2 i}\left[A(t) B(t)-A^{\dagger}(t) B^{\dagger}(t)\right] .
\end{aligned}
$$

Under short-time approximation, we keep terms up to first order in " $g t$ " in Taylor's expansion to get

$$
\begin{aligned}
& A(t)=A(0)+t \dot{A}(0)+\ldots \ldots, \\
& B(t)=B(0)+t \dot{B}(0)+\ldots \ldots
\end{aligned}
$$

Using equations (31) and (32) in equations (14)-(17) gives

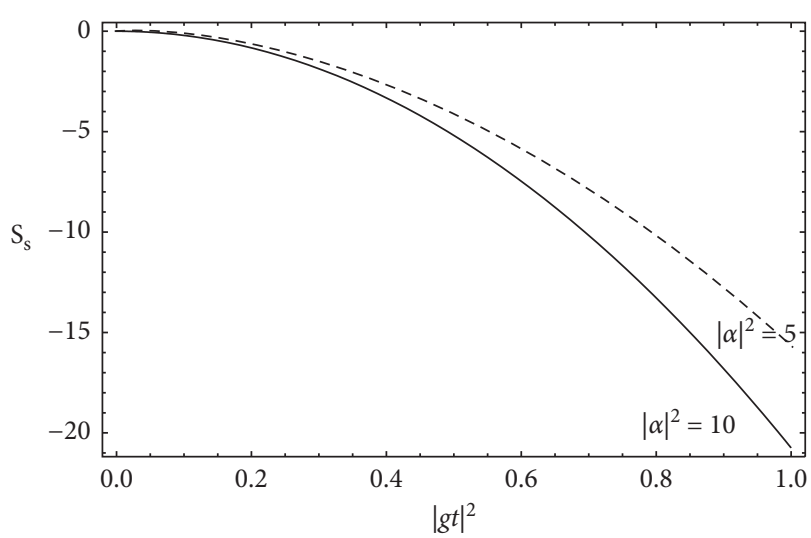

FIgURE 2: Variation of the sum squeezing $S_{\mathrm{S}}$ with $|g t|^{2}$ in the nondegenerate frequency upconversion process (when $\left\langle N_{B}\right\rangle=$ $\left.|\beta|^{2}=10\right)$.

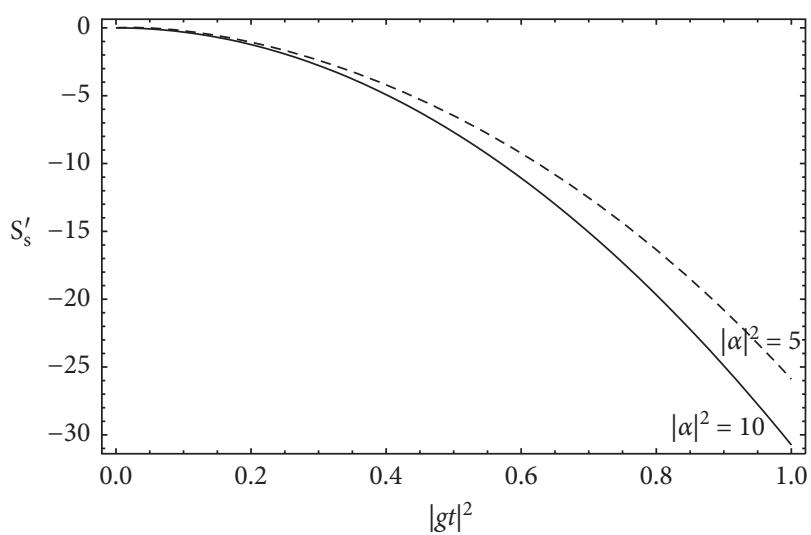

FIGURE 3: Variation of the sum squeezing $S_{S}^{\prime}$ with $|g t|^{2}$ in the nondegenerate frequency upconversion process (when $\left\langle N_{B}\right\rangle=$ $\left.|\beta|^{2}=20\right)$.

$$
\begin{gathered}
A(t)=A-i g t B^{\dagger} C, \\
A^{\dagger}(t)=A^{\dagger}+i g t B C^{\dagger}, \\
B(t)=B-i g t A^{\dagger} B, \\
B^{\dagger}(t)=B^{\dagger}+i g t A B^{\dagger} .
\end{gathered}
$$

Using equations (33)-(36) in equation (29), we find $W_{1 C}(t)=\frac{1}{2}\left[\left(A B+A^{\dagger} B^{\dagger}\right)-i g t\left\{B^{\dagger} B\left(C-C^{\dagger}\right)+A^{\dagger} A\left(B-B^{\dagger}\right)\right\}\right]$.

As the wave function of the system starts as a coherent state at $t=0$ and evolves as a squeezed state at ater time [46], we use the initial coherent state.

Now, initially we consider a quantum state as a product of coherent states $\mid \alpha>$ and $\mid \beta>$ for the pump modes $A$ and $B$, respectively, and $|\gamma\rangle$ for the sum-frequency mode $C$, i.e., 


$$
|\psi\rangle=|\alpha\rangle_{A}|\beta\rangle_{B}|\gamma\rangle_{C}
$$

Using equation (38) in equation (37), we obtain

$$
\begin{aligned}
\left\langle\psi\left|W_{1 C}^{2}\right| \psi\right\rangle= & \frac{1}{4}\left[\alpha^{2} \beta^{2}+\alpha^{* 2} \beta^{* 2}+2|\alpha|^{2}|\beta|^{2}+|\alpha|^{2}+|\beta|^{2}+1-i g t\left(2 \alpha \beta^{*}|\beta|^{2} \gamma-2 \alpha \beta^{*}|\beta|^{2} \gamma^{*}+2 \alpha|\alpha|^{2} \beta^{2}-2 \alpha|\alpha|^{2}|\beta|^{2}\right.\right. \\
& +2 \alpha^{*} \beta^{*} \gamma|\beta|^{2}-2 \alpha^{*} \beta^{*} \gamma^{*}|\beta|^{2}+2 \alpha^{*}|\beta|^{2}|\alpha|^{2}-2 \alpha^{*} \beta^{* 2}|\alpha|^{2}+\alpha \beta \gamma-\alpha \beta \gamma^{*}+\alpha \beta^{2}-\alpha^{*} \beta^{* 2}-\alpha|\beta|^{2}+\alpha^{*}|\beta|^{2} \\
& \left.\left.-\alpha|\alpha|^{2}+\alpha^{*}|\alpha|^{2}+\alpha^{*} \beta^{*} \gamma-\alpha^{*} \beta^{*} \gamma^{*}-\alpha+\alpha^{*}\right)\right] \\
\left\langle\psi\left|W_{1 C}\right| \psi\right\rangle^{2}= & \frac{1}{4}\left[\alpha^{2} \beta^{2}+\alpha^{* 2} \beta^{* 2}+2|\alpha|^{2}|\beta|^{2}-i g t\left(2 \alpha \beta^{*}|\beta|^{2} \gamma-2 \alpha \beta^{*}|\beta|^{2} \gamma^{*}+2 \alpha|\alpha|^{2} \beta^{2}-2 \alpha|\alpha|^{2}|\beta|^{2}\right.\right. \\
& \left.\left.+2 \alpha^{*} \beta^{*} \gamma|\beta|^{2}-2 \alpha^{*} \beta^{*} \gamma^{*}|\beta|^{2}+2 \alpha^{*}|\beta|^{2}|\alpha|^{2}-2 \alpha^{*} \beta^{* 2}|\alpha|^{2}\right)\right] \\
{\left[\Delta W_{1 C}(t)\right]^{2}=} & \left\langle W_{1 C}^{2}(t)\right\rangle-\left\langle W_{1 C}(t)\right\rangle^{2} \\
= & \frac{1}{4}\left[|\alpha|^{2}+|\beta|^{2}+1-i g t\left(\alpha \beta \gamma-\alpha \beta \gamma^{*}+\alpha \beta^{2}-\alpha^{*} \beta^{* 2}-\alpha|\beta|^{2}+\alpha^{*}|\beta|^{2}-\alpha|\alpha|^{2}+\alpha^{*}|\alpha|^{2}+\alpha^{*} \beta^{*} \gamma\right.\right. \\
& \left.\left.-\alpha^{*} \beta^{*} \gamma^{*}-\alpha+\alpha^{*}\right)\right]
\end{aligned}
$$

The numbers of photons are

$$
\begin{aligned}
& N_{A}(t)=A^{\dagger}(t) A(t)=N_{A}+i g t\left(A B C^{\dagger}-A^{\dagger} B^{\dagger} C\right), \\
& N_{B}(t)=B^{\dagger}(t) B(t)=N_{B}+i g t\left(A B C^{\dagger}-A^{\dagger} B^{\dagger} C\right) .
\end{aligned}
$$

Using equation (38), we obtain

$$
\begin{aligned}
\frac{1}{4}\left\langle N_{A}(t)+N_{B}(t)+1\right\rangle= & \frac{1}{4}\left[|\alpha|^{2}+|\beta|^{2}+1\right. \\
& \left.+2 i g t\left(\alpha \beta \gamma^{*}-\alpha^{*} \beta^{*} \gamma\right)\right] .
\end{aligned}
$$

Subtraction of equation (44) from equation (41) yields

$$
\begin{aligned}
{[} & \left.\Delta W_{1 C}(t)\right]^{2}-\frac{1}{4}\left\langle N_{A}(t)+N_{B}(t)+1\right\rangle \\
& =\frac{-i g t}{4}\left[\left(\alpha \beta-\alpha^{*} \beta^{*}\right)\left(\gamma+\gamma^{*}\right)+\left(\alpha \beta^{2}-\alpha^{*} \beta^{* 2}\right)+\left(|\alpha|^{2}+|\beta|^{2}+1\right)\left(\alpha^{*}-\alpha\right)\right] \\
& =\frac{g t}{2}\left[2|\alpha \beta \gamma| \sin \left(\theta_{1}+\theta_{2}\right) \cos \theta_{3}+\left|\alpha \beta^{2}\right| \sin \left(\theta_{1}+2 \theta_{2}\right)-\left(|\alpha|^{2}+|\beta|^{2}+1\right)|\alpha| \sin \theta_{1}\right],
\end{aligned}
$$

where $\alpha=|\alpha| \exp \left(i \theta_{1}\right), \beta=|\beta| \exp \left(i \theta_{2}\right)$, and $\gamma=|\gamma| \exp \left(i \theta_{3}\right)$.

Equation (45) means that squeezing of $W_{1 C}$ will occur whenever $\theta_{1}$ and $\theta_{2}<0$ and $\theta_{3}>0$. It suffices to choose $\theta_{1}=\theta_{2}=-(\pi / 3)$ and $\theta_{3}=(\pi / 2)$, and the square bracket becomes nonnegative.
Let us now study the dependence of sum squeezing for two-mode states on squeezing of individual modes in which the modes are uncorrelated, i.e., mode in a coherent state at $t=0$.

We define for two-mode sum squeezing as [34] 


$$
W_{C \varphi}=\frac{1}{2}\left(e^{i \varphi} A^{\dagger} B^{\dagger}+e^{-i \varphi} A B\right) .
$$

The squeezed state exists if $\Delta W_{C \varphi}<(1 / 2)$ for some $\varphi$. Using equation (46), we obtain

$$
\begin{aligned}
\left\langle W_{C \varphi}^{2}\right\rangle & =\frac{1}{4}\left\langle e^{2 i \varphi}\left(A^{\dagger} B^{\dagger}\right)^{2}+A^{\dagger} B^{\dagger} A B+A B A^{\dagger} B^{\dagger}+e^{-2 i \varphi}(A B)^{2}\right\rangle \\
& =\frac{1}{4}\left\langle e^{2 i \varphi}\left(A^{\dagger} B^{\dagger}\right)^{2}+2 B^{\dagger} B A^{\dagger} A+A^{\dagger} A+B^{\dagger} B+1+e^{-2 i \varphi}(A B)^{2}\right\rangle, \\
\left\langle W_{C \varphi}\right\rangle^{2} & =\frac{1}{4}\left[\left\langle e^{2 i \varphi}\left\langle A^{\dagger} B^{\dagger}\right\rangle^{2}+2\left\langle A^{\dagger} B^{\dagger}\right\rangle\langle A B\rangle+e^{-2 i \varphi}\langle A B\rangle^{2}\right\rangle\right] .
\end{aligned}
$$

Hence, the variance of field is

$$
\begin{aligned}
{\left[\Delta W_{C \varphi}\right]^{2}=} & \left\langle W_{C \varphi}^{2}\right\rangle-\left\langle W_{C \varphi}\right\rangle^{2} \\
= & \frac{1}{4}\left\{e^{2 i \varphi}\left[\left\langle\left(A^{\dagger} B^{\dagger}\right)^{2}\right\rangle-\left\langle A^{\dagger} B^{\dagger}\right\rangle^{2}\right]+2\left\langle B^{\dagger} B A^{\dagger} A\right\rangle+\left\langle B^{\dagger} B\right\rangle+\left\langle A^{\dagger} A\right\rangle+1\right. \\
& \left.-2\left\langle A^{\dagger} B^{\dagger}\right\rangle\langle A B\rangle+e^{-2 i \varphi}\left[\left\langle(A B)^{2}\right\rangle-\langle A B\rangle^{2}\right]\right\} .
\end{aligned}
$$

Using equation (9) in equation (49), we find

$$
\begin{aligned}
{\left[\Delta W_{C \varphi}\right]^{2}-\frac{1}{4}\left\langle N_{A}+N_{B}+1\right\rangle=} & \frac{1}{4}\left\{e^{2 i \varphi}\left[\left\langle\left(A^{\dagger} B^{\dagger}\right)^{2}\right\rangle-\left\langle\left(A^{\dagger} B^{\dagger}\right)\right\rangle^{2}\right]+2\left\langle B^{\dagger} B A^{\dagger} A\right\rangle\right. \\
& \left.-2\left\langle A^{\dagger} B^{\dagger}\right\rangle\langle A B\rangle+e^{-2 i \varphi}\left[\left\langle(A B)^{2}\right\rangle-\langle A B\rangle^{2}\right]\right\} .
\end{aligned}
$$

A state is squeezed if the term in brackets becomes negative. This term is smallest when

$$
\arg \left[\left\langle(A B)^{2}\right\rangle-\langle A B\rangle^{2}\right]-2 \varphi=\pi .
$$

If $\varphi$ satisfies (51), then

$$
\begin{aligned}
{\left[\Delta W_{C \varphi}\right]^{2}-\frac{1}{4}\left\langle N_{A}+N_{B}+1\right\rangle=} & \frac{1}{2}\left[\left\langle N_{A} N_{B}\right\rangle-|\langle A B\rangle|^{2}\right. \\
& \left.-\left|\left\langle(A B)^{2}\right\rangle-\langle A B\rangle^{2}\right|\right] .
\end{aligned}
$$

Therefore, a state is sum squeezed if and only if

$$
\left|\left\langle(A B)^{2}\right\rangle-\langle A B\rangle^{2}\right|>\left\langle\left(N_{A} N_{B}\right)\right\rangle-|\langle(A B)\rangle|^{2} .
$$

If the modes are uncorrelated, then equation (53) becomes

$$
\left.\left|\left\langle A^{2}\right\rangle\left\langle B^{2}\right\rangle-\langle A\rangle^{2}\langle B\rangle^{2}\right|\right\rangle\left\langle N_{A}\right\rangle\left\langle N_{B}\right\rangle-|\langle A\rangle\langle B\rangle|^{2} .
$$

Let us consider the first case. If the modes are uncorrelated, i.e., there is no linear relationship between $A$ and $B$ or $\langle A\rangle=\langle B\rangle=0$ and neither the $A$ nor the $B$ mode is squeezed [34], then

$$
\begin{gathered}
\left|\left\langle A^{2}\right\rangle-\langle A\rangle^{2}\right| \leq\left\langle N_{A}\right\rangle-|\langle A\rangle|^{2}, \\
\left|\left\langle B^{2}\right\rangle-\langle B\rangle^{2}\right| \leq\left\langle N_{B}\right\rangle-|\langle B\rangle|^{2} .
\end{gathered}
$$

Furthermore, if none of the $A$ and $B$ modes are squeezed, i.e., at coherent state, none of the pairs can be sum squeezed [34], i.e.,

$$
\left|\left\langle A^{2}\right\rangle\left\langle B^{2}\right\rangle-\langle A\rangle^{2}\langle B\rangle^{2}\right| \leq\left\langle N_{A}\right\rangle\left\langle N_{B}\right\rangle-|\langle A\rangle\langle B\rangle|^{2} .
$$

Comparing equations (54) and (57), we find that the $A$ and $B$ modes are not sum squeezed.

In the second case, if the $A$ mode is squeezed and the $B$ mode is in a coherent state of amplitude $\beta$, then we have 


$$
\begin{aligned}
\left|\left\langle A^{2}\right\rangle\left\langle B^{2}\right\rangle-\langle A\rangle^{2}\langle B\rangle^{2}\right| & =|\beta|^{2}\left|\left(\left\langle A^{2}\right\rangle-\langle A\rangle^{2}\right)\right| \\
& >|\beta|^{2}\left(\left\langle N_{A}\right\rangle-|\langle A\rangle|^{2}\right),
\end{aligned}
$$

where $\left\langle N_{B}\right\rangle=|\beta|^{2}$ for coherent states and the inequality in equation (54) is fulfilled; hence, state is sum squeezed.

In the third case, if the $B$ mode is squeezed and the $A$ mode is in a coherent state of amplitude $\alpha$, we then have

$$
\begin{aligned}
\left|\left\langle A^{2}\right\rangle\left\langle B^{2}\right\rangle-\langle A\rangle^{2}\langle B\rangle^{2}\right| & =|\alpha|^{2}\left|\left(\left\langle B^{2}\right\rangle-\langle B\rangle^{2}\right)\right| \\
& >|\alpha|^{2}\left(\left\langle N_{B}\right\rangle-|\langle B\rangle|^{2}\right),
\end{aligned}
$$

where $\left\langle N_{A}\right\rangle=|\alpha|^{2}$ and the inequality in equation (54) is satisfied hence the $A$ and $B$ modes are sum squeezed.

Finally, if $A$ and $B$ modes are squeezed, then we have

$$
\left.\left|\left\langle A^{2}\right\rangle\left\langle B^{2}\right\rangle-\langle A\rangle^{2}\langle B\rangle^{2}\right|\right\rangle\left\langle N_{A}\right\rangle\left\langle N_{B}\right\rangle-|\langle A\rangle\langle B\rangle|^{2} \text {. }
$$

This satisfies condition (54) and hence the state is sum squeezed.

\section{Sum Squeezing in Degenerate Frequency Upconversion Process}

If all the two pump modes are of the same frequency $\left(\omega_{a}=\omega_{b}\right)$, the process reduces to second harmonic generation described by the Hamiltonian $H^{\prime}$ given by

$$
H^{\prime}=\omega_{a} a^{\dagger} a+\omega_{c} c^{\dagger} c+g\left(a^{2} c^{\dagger}+a^{\dagger 2} c\right) \text {. }
$$

Equation (15) leads to coupled Heisenberg equations of motion:

$$
\begin{aligned}
& \dot{A}=-2 i g A^{\dagger} C, \\
& \dot{C}=-i g A^{2} .
\end{aligned}
$$
obtain

Using Taylor's expansion upto second order in $g t$, we

$$
\begin{gathered}
C(t)=C-i g t A^{2}-g^{2} t^{2}\left(2 A^{\dagger} A+1\right) C, \\
C^{\dagger}(t)=C+i g t A^{\dagger 2}-g^{2} t^{2}\left(2 A^{\dagger} A+1\right) C^{\dagger} .
\end{gathered}
$$

Using equations (20) and (21), this gives

$$
\begin{aligned}
& X_{1 C}(t)=X_{1 C}+g t Y_{2}-4 g^{2} t^{2}\left(N_{A}+\frac{1}{2}\right) X_{1 C}, \\
& X_{2 C}(t)=X_{2 C}-g t Y_{1}-4 g^{2} t^{2}\left(N_{A}+\frac{1}{2}\right) X_{2 C} .
\end{aligned}
$$

If the $C$ mode is initially in a coherent state at $t=0$, then we obtain

$$
\begin{aligned}
& {\left[\Delta X_{1 C}(t)\right]^{2}-\frac{1}{4}=|g t|^{2}\left[\left(\Delta Y_{2}\right)^{2}-\left\langle N_{A}+\frac{1}{2}\right\rangle\right],} \\
& {\left[\Delta X_{2 C}(t)\right]^{2}-\frac{1}{4}=|g t|^{2}\left[\left(\Delta Y_{1}\right)^{2}-\left\langle N_{A}+\frac{1}{2}\right\rangle\right] .}
\end{aligned}
$$

From equations (67) and (68), we infer that $X_{1 C}$ becomes squeezed if $Y_{2}$ is squeezed and $X_{2 C}$ is squeezed if $Y_{1}$ is squeezed. In other way, the $C$ mode is squeezed in the $X_{1 C}$

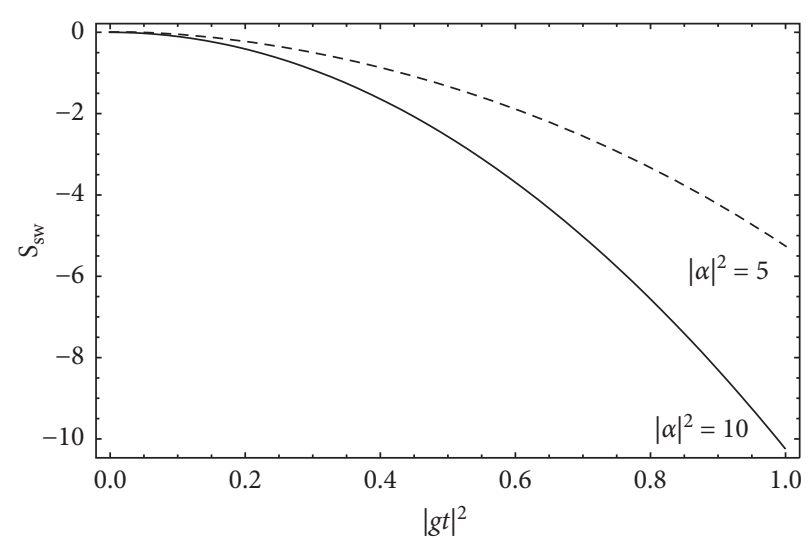

FIgURE 4: Variation of the sum squeezing $S_{\mathrm{SW}}$ with $|g t|^{2}$ in the degenerate frequency upconversion process.

direction if the fundamental modes are sum squeezed in the $Y_{2}$ direction and the $C$ mode is squeezed in the $X_{2 C}$ direction if the fundamental modes are sum squeezed in the $Y_{1}$ direction. These equations show that the amplitude-squared squeezing of the fundamental can be turned directly into the squeezing of the harmonic in the degenerate frequency upconversion process. This result suggests a way to detect higher-order squeezing in this process.

We plot a graph (Figure 4) between left-hand side of equation (67) or (68) say $S_{\mathrm{SW}}$ and $|g t|^{2}$ with typical values $\left(\Delta Y_{2}\right)^{2}=\left(\Delta Y_{1}\right)^{2}=(1 / 4)$ so that it could satisfy equation (4).

Figure 4 shows that the plot responses nonlinearly to increase photon numbers. This confirms that the squeezed states are associated with large number of pump photons. It also confirms that the higher-order squeezing (sum squeezing) is directly associated with the coupling of the field and interaction time. Hence, optimum squeezing can be realized in short-time scale.

\section{Conclusions}

In this paper, we have concluded that sum squeezing can be turned into normal squeezing via sum-frequency generation in the nondegenerate frequency upconversion process. It is also established that the amplitude-squared (higher-order) squeezing of the fundamental can be converted directly into the squeezing of the harmonic in the degenerate frequency upconversion process. These findings suggest a method for generation and detection of higher-order squeezing in the frequency upconversion process. It is observed that the sum squeezing will occur conditional to the case of first-order coupling, while to the case of second-order coupling gives unconditional sum squeezing in nondegenerate as well as in the degenerate frequency upconversion process.

We have examined all possible conditions for an uncorrelated two-mode state. If both modes are not squeezed, then the state is not sum squeezed. If one mode is squeezed and the second one is in a coherent state, then the state is sum squeezed. Finally, if both modes are squeezed, then the state may or may not be sum squeezed in the nondegenerate frequency upconversion process. The steady fall of the curves infers that the sum squeezing exists and responses 
nonlinearly to the number of pump photons. It shows that the squeezed states are associated with large number of pump photons. It also confirms that the higher-order squeezing (sum squeezing) is directly associated with the coupling of the field and interaction time. Hence, optimum squeezing can be realized in short-time scale. It is found that the depth of nonclassicality is increasing with an increase of the number of pump photons. Hence, it is inferred that a higher multiphoton absorption process is suitable for generation of optimum squeezed light.

These findings suggest and may help in selecting a suitable process to generate optimum squeezing of the radiation and further can be useful as a resource to improve high-quality optical telecommunication [47]. The results obtained in this paper are of interest for new experiments on the study of nonlinear optical processes in dielectric media using ultrashort intense laser pulses as exciting radiation, and the effects of damping and decoherence as well as higher-order time terms could be investigated.

\section{Data Availability}

The data used to support the findings of this study are available from the corresponding author upon request.

\section{Conflicts of Interest}

The authors declare that they have no conflicts of interest.

\section{References}

[1] D. F. Walls, "Squeezed states of light," Nature, vol. 306, no. 5939, pp. 141-146, 1983.

[2] R. Loudon and P. L. Knight, "Squeezed light," Journal of Modern Optics, vol. 34, no. 6-7, pp. 709-759, 1987.

[3] M. C. Teich and B. E. A. Saleh, "Squeezed state of light," Quantum Optics: Journal of the European Optical Society Part $B$, vol. 1, no. 2, pp. 153-191, 1989.

[4] J. Perina, Quantum Statistics of Linear and Nonlinear Optical Phenomena, Chapters 9, 10 and 11, Kluwer, Dordrecht, Netherlands, 1991.

[5] L. Mandel, "Non-Classical states of the electromagnetic field," Physica Scripta, vol. T12, pp. 34-42, 1986.

[6] V. V. Dodonov, "Nonclassical' states in quantum optics: a 'squeezed' review of the first 75 years," Journal of Optics B: Quantum and Semiclassical Optics, vol. 4, no. 1, pp. R1-R33, 2002.

[7] B. E. A. Saleh and M. C. Teich, "Can the channel capacity of a light-wave communication system be increased by the use of photon-number-squeezed light?," Physical Review Letters, vol. 58, no. 25, pp. 2656-2659, 1987.

[8] K. Wódkiewicz, "On the quantum mechanics of squeezed states," Journal of Modern Optics, vol. 34, no. 6-7, pp. 941-948, 1987.

[9] H. J. Kimble and D. F. Walls, "Introduction," Journal of the Optical Society of America B, vol. 4, no. 10, pp. 1450-1741, 1987.

[10] H. Yuen and J. Shapiro, "Optical communication with twophoton coherent states-part I: quantum-state propagation and quantum-noise," IEEE Transactions on Information Theory, vol. 24, no. 6, pp. 657-668, 1978.
[11] C. H. Bennett, G. Brassard, and N. D. Mermin, "Quantum cryptography without Bell's theorem," Physical Review Letters, vol. 68, no. 5, pp. 557-559, 1992.

[12] J. Kempe, "Multiparticle entanglement and its applications to cryptography," Physical Review A, vol. 60, no. 2, pp. 910-916, 1999.

[13] L. Mandel, "Squeezing and photon antibunching in harmonic generation," Optics Communications, vol. 42, no. 6, pp. 437439, 1982.

[14] M. Hillery, "Squeezing of the square of the field amplitude in second harmonic generation," Optics Communications, vol. 62, no. 2, pp. 135-138, 1987.

[15] S. Kielich, R. Tanaś, and R. Zawodny, "Squeezing in the thirdharmonic field generated by self-squeezed light," Journal of the Optical Society of America B, vol. 4, no. 10, pp. 1627-1632, 1987.

[16] Y.-B. Zhan, "Amplitude-cubed squeezing in harmonic generations," Physics Letters A, vol. 160, no. 6, pp. 498-502, 1991.

[17] J. Perina, V. Perinova, C. Sibilia, and M. Bertolotti, "Quantum statistics of four-wave mixing," Optics Communications, vol. 49, no. 4, pp. 285-289, 1984.

[18] M. S. K. Razmi and J. H. Eberly, "Degenerate four-wave mixing and squeezing in pumped three-level atomic systems," Optics Communications, vol. 76, no. 3-4, pp. 265-267, 1990.

[19] D. K. Giri and P. S. Gupta, "The squeezing of radiation in fourwave mixing processes," Journal of Optics B: Quantum and Semiclassical Optics, vol. 6, no. 1, pp. 91-96, 2004.

[20] D. K. Giri and P. S. Gupta, "Short-time squeezing effects in spontaneous and stimulated six-wave mixing process," Optics Communications, vol. 221, no. 1-3, pp. 135-143, 2003.

[21] J. Perina and J. Krepelka, "Stimulated Raman scattering of squeezed light with pump depletion," Journal of Modern Optics, vol. 38, no. 5, pp. 2137-2151, 1992.

[22] J. Perina and J. Krepelka, "Quantum statistics of stimulated Raman and hyper-Raman scattering of squeezed light with pump depletion," Journal of Modern Optics, vol. 39, no. 5, pp. 1029-1041, 1992.

[23] A. Kumar and P. S. Gupta, "Short-time squeezing in spontaneous Raman and stimulated Raman scattering," Quantum and Semiclassical Optics: Journal of the European Optical Society Part B, vol. 7, no. 5, pp. 835-841, 1995.

[24] D. K. Giri and P. S. Gupta, "Higher-order squeezing of the electromagnetic field in spontaneous and stimulated Raman processes," Journal of Modern Optics, vol. 52, no. 12, pp. 1769-1781, 2005.

[25] A. Kumar and P. S. Gupta, "Higher-order amplitude squeezing in hyper-Raman scattering under short-time approximation," Quantum and Semiclassical Optics: Journal of the European Optical Society Part B, vol. 8, no. 5, pp. 10531060, 1996.

[26] C. K. Hong and L. Mandel, "Higher-order squeezing of a quantum field," Physical Review Letters, vol. 54, no. 4, pp. 323-325, 1985.

[27] C. K. Hong and L. Mandel, "Generation of higher-order squeezing of quantum electromagnetic fields," Physical Review A, vol. 32, no. 2, pp. 974-982, 1985.

[28] M. Hillery, "Amplitude-squared squeezing of the electromagnetic field," Physical Review A, vol. 36, no. 8, pp. 37963802, 1987.

[29] M. Hillery, "Phase-space representation of amplitude-squared squeezing," Physical Review A, vol. 45, no. 7, pp. 4944-4950, 1992.

[30] J. Perina, V. Perinova, and J. Kodousek, "On the relations of antibunching, sub-poissonian statistics and squeezing," Optics Communications, vol. 49, no. 3, pp. 210-214, 1984. 
[31] Y. Kim and T. H. Yoon, "Higher order sub-poissonian photon statistics of light," Optics Communications, vol. 212, no. 1-3, pp. 107-114, 2002.

[32] H. Prakash and D. K. Mishra, "Higher order sub-poissonian photon statistics and their use in detection of Hong and Mandel squeezing and amplitude-squared squeezing," Journal of Physics B: Atomic, Molecular and Optical Physics, vol. 39, no. 9, pp. 2291-2297, 2006.

[33] D. K. Mishra, "Study of higher order non-classical properties of squeezed Kerr state," Optics Communications, vol. 283, no. 17 , pp. 3284-3290, 2010.

[34] M. Hillery, "Sum and difference squeezing of the electromagnetic field," Physical Review A, vol. 40, no. 6, pp. 31473155, 1989.

[35] A. Kumar and P. S. Gupta, "Sum squeezing in four-wave sum frequency generation," Optics Communications, vol. 136, no. 5-6, pp. 441-446, 1997.

[36] A. Kumar and P. S. Gupta, "Difference squeezing in four-wave difference-frequency generation," Quantum and Semiclassical Optics: Journal of the European Optical Society Part B, vol. 10, no. 3, pp. 485-492, 1998.

[37] D. K. Giri and P. S. Gupta, "Squeezing effects in the sum and difference of the field amplitude in the Raman process," Modern Physics Letters B, vol. 19, no. 25, pp. 1261-1276, 2005.

[38] M. K. Olsen and R. J. Horowicz, "Squeezing in the sum and difference fields in second harmonic generation," Optics Communications, vol. 168, no. 1-4, pp. 135-143, 1999.

[39] N. B. An and T. Vo, "General multimode sum-squeezing," Physics Letters A, vol. 261, no. 1-2, pp. 34-39, 1999.

[40] N. B. An and T. Tinh, "General multimode differencesqueezing," Physics Letters A, vol. 270, no. 1-2, pp. 27-40, 2000.

[41] R. Prakash and P. Shukla, "Detection of sum and difference squeezing," IOSR Journal of Applied Physics, vol. 1, no. 2, pp. 43-47, 2012.

[42] A. Mukherjee, A. Giri, B. Sarkar, and B. Sen, "Sum and difference squeezing in second harmonic generation," International Research Journal of Basic and Applied Sciences, vol. 1, pp. 39-42, 2016.

[43] K. Mukherjee and P. C. Jana, "Higher-order squeezing of a generic quadratically-coupled optomechanical system," IOSR Journal of Applied Physics, vol. 9, no. 1, pp. 90-95, 2017.

[44] L. Ma, O. Slattery, and X. Tang, "Single photon frequency upconversion and its applications," Physics Reports, vol. 521, no. 2, pp. 69-94, 2012.

[45] R. Tanas, "Squeezing from an anharmonic oscillator model: $\left(a^{\dagger}\right)^{2} a^{2}$ versus $\left(a^{\dagger} a\right)^{2}$ interaction Hamiltonian," Physics Letters A, vol. 141, no. 5-6, pp. 217-220, 1989.

[46] C. F. Lo, "Time evolution of a charged oscillator with a timedependent mass and frequency in a time-dependent electromagnetic field," Physical Review A, vol. 45, no. 7, pp. 5262-5265, 1992.

[47] D. K. Giri, R. P. Singh, and A. Bandyopadhyay, "Displacement gain dependent fidelity in quantum teleportation using entangled two-mode squeezed light," Optical and Quantum Electronics, vol. 46, no. 9, pp. 1127-1137, 2014. 